\title{
A randomized comparison of 5 versus 12 hours per day of cardiac contractility modulation treatment for heart failure patients: A preliminary report
}

\author{
Axel Kloppe ${ }^{1}$, Dejan Mijic ${ }^{2}$, Fabian Schiedat ${ }^{1}$, Harilaos Bogossian $^{2}$, \\ Andreas Mügge ${ }^{1}$, Benny Rousso ${ }^{3}$, Bernd Lemke ${ }^{2}$ \\ ${ }^{1}$ Department of Cardiology and Angiology, Klinikum Bergmannsheil, Ruhr University Bochum, Germany \\ ${ }^{2}$ Department of Cardiology and Angiology, Klinikum Luedenscheid, Luedenscheid, Germany \\ ${ }^{3}$ Impulse Dynamics, Hod Hasharon, Israel
}

\begin{abstract}
Background: Cardiac contractility modulation (CCM) signals are non-excitatory electrical signals delivered during the absolute refractory period intended to improve contraction and cardiac function. Clinical trials have shown that CCM treatment significantly improves exercise tolerance and quality of life in symptomatic heart failure patients. Studies with CCM therapy typically include CCM delivery for 3, 5 or $7 \mathrm{~h}$ per day, although other configurations are also commonly used. Each has been associated with improved outcomes in heart failure, but it is not clear whether different application durations are associated with the various degrees of benefit. The purpose of the current pilot evaluation study was to evaluate the quality of life, exercise tolerance, and cardiac function, over a 6-month period when CCM was delivered for $5 \mathrm{~h} /$ day vs. $12 \mathrm{~h} /$ day. Increasing the daily CCM therapy duration is safe and as good as the standard CCM periods of application per day.
\end{abstract}

Methods: This single center pilot evaluation study involved 19 medically refractory symptomatic patients with heart failure and reduced left ventricular function who underwent implantation of an Optimizer ${ }^{\mathrm{TM}}$ system (Impulse Dynamics, Orangeburg, NY, USA). Patients were randomized into one of two treatment groups; $5 \mathrm{~h} /$ day CCM treatment or $12 \mathrm{~h} /$ day CCM treatment. Subjects and evaluating physicians were blinded to the study group. Subjects returned to the hospital after 12 and 24 weeks. Efficacy evaluations included changes from baseline to 24 weeks in Minnesota Living With Heart Failure Questionnaire score (MLWHFQ), maximal oxygen consumption in the cardio-pulmonary stress test (peak $\mathrm{VO}_{2}$ ), New York Heart Association classification (NYHA), 6-min walk distance (6MWD), and ejection fraction (EF).

Results: At the end of 24 weeks, clinical improvement was observed in the entire cohort in all efficacy measures (mean change from baseline of -17.1 in MLWHFQ, -0.86 in NYHA, and improvement trend of $1.48 \mathrm{~mL} \mathrm{O}_{2} / \mathrm{kg} / \mathrm{min}$ in peak $\mathrm{VO}_{2}, 31.3 \mathrm{~m}$ in $6 \mathrm{MWD}$, and $2.25 \%$ in $\mathrm{EF}$ ). There were no significant differences, either clinically or statistically, between the groups receiving CCM for $5 \mathrm{~h} /$ day vs. $12 \mathrm{~h} /$ day. Three subjects were voluntarily withdrawn before completing the study. One subject died from pneumonia after 125 days, and 6 serious adverse events were reported, none of which was classified as related to either the device or the procedure.

Conclusions: Together with previously reported experience with CCM, delivery of CCM therapy is equally safe and appears similarly effective over the range of shorter (5 h) to longer (12 h) daily periods of application. Given the small sample size, further studies are warranted. (Cardiol J 2016; 23, 1: 114-119)

Key words: cardiac contractility modulation, heart failure, electrical treatment of heart failure

Address for correspondence: Axel Kloppe, MD, Klinik für Kardiologie und Angiologie, Medizinische Klinik II,

Berufsgenossenschaftliches Universitätsklinikum, Bergmannsheil GmbH, Bürkle-de-la-Camp-Platz 1, 44789 Bochum, Germany, tel: +49-234 3026060, fax: +49-234 3026051, e-mail: kloppe@web.de 


\section{Introduction}

Cardiac contractility modulation (CCM) is the application of non-excitatory electrical signals to the myocardium, during the absolute refractory period of the action potential, to augment contraction, and to promote reverse remodeling of heart failure (HF). This effect appears to contribute to long-term clinical benefit to HF patients, and is achieved without increasing myocardial oxygen consumption [1].

The bases for the effects of CCM have been reviewed recently [2]. The action of CCM appears to be mediated by molecular mechanisms that restore the ability of failing myocytes to more normally handle calcium cycling. This ameliorative action includes phosphorylation of cardiac phospholamban which acutely improves the ability of the sarcoplasmic reticulum to sequester calcium. Chronically, CCM stimulates gene expression that restores production of proteins involved in calcium handling and contraction.

Following two pilot studies of chronic CCM signal application $[3,4]$ that demonstrated the device safety and functionality, larger scale studies were performed.

In a study of 428 patients with New York Heart Association (NYHA) class III or IV, narrow QRS HF (FIX-HF-5), CCM was found to be safe and showed an effect of improving quality of life and raising maximal oxygen consumption in cardio-pulmonary stress test (peak $\mathrm{VO}_{2}$ ) compared with the control group [5].

Subsequent analysis of patient subgroups revealed that CCM improves multiple objective parameters of exercise tolerance, including ventilatory anaerobic threshold, in a subgroup of patients characterized by normal QRS duration, NYHA functional class III symptoms, and ejection fraction (EF) of at least 25\% [6].

Although previous studies have demonstrated the safety and efficacy of $3 \mathrm{~h} \mathrm{[4],5} \mathrm{h} \mathrm{[6]} \mathrm{and} 7 \mathrm{~h} \mathrm{[7]}$ of CCM therapy per day, no direct comparison of the effects of different durations of CCM stimulation have been reported.

The objective of this pilot evaluation study was to compare the impact of 6 months of $5 \mathrm{~h}$ vs. $12 \mathrm{~h}$ of CCM therapy per day on symptoms, quality of life and exercise tolerance in patients with medically refractory symptomatic $\mathrm{HF}$ and reduced left ventricular (LV) function.

\section{Methods}

\section{Patients}

This double blind active control study included 19 patients. All procedures were approved by the local Ethics Committee and informed consent was obtained from all enrollees. Eligibility included: being above 18 years of age; stable use of optimal medical therapy for HF with doses and duration of treatment based on institutional standards of care; LVEF of $35 \%$ or less by echocardiography; and NYHA class III or IV symptoms.

The major exclusion criteria included: permanent atrial fibrillation, peak $\mathrm{VO}_{2}<9 \mathrm{~mL} \mathrm{O} /$ $/ \mathrm{kg} / \mathrm{min}$, myocardial infarction within 3 months of enrollment, mechanical tricuspid or aortic valves, hospitalization for HF less than 2 weeks before enrollment and requiring the use of intravenous diuretics or inotropic support; reduced exercise tolerance for reasons other than HF (e.g., angina, chronic obstructive pulmonary disease, peripheral vascular disease, orthopedic or rheumatologic conditions).

After obtaining informed consent, enrolled subjects were evaluated by echocardiography, cardiopulmonary stress testing, a 6-min walk distance (6MWD), HF questionnaire, and a 24-h Holter monitor.

Table 1 lists the baseline demographics and characteristics of participants. No statistical difference was apparent between the groups.

\section{Study design}

Following Optimizer implantation subjects were randomized to one of two treatment groups: group A ( $5 \mathrm{~h} /$ day CCM treatment) or group B (12 h/day CCM treatment), and the Optimizer pulse generator was programmed accordingly prior to hospital discharge. The CCM delivery schedule was programmed for multiple equally spaced stimuli of $1 \mathrm{~h}$ treatments.

Primary follow-up visits were performed at week 24 with interrogation of implanted devices (CCM, ICD). A medical history including NYHA classification and medications, physical examination, Minnesota Living Heart Failure Questionnaire (MLWHFQ) and 6MWD were obtained along with a cardiopulmonary stress test and echocardiogram.

\section{Statistical methods}

This study was a pilot evaluation of $5 \mathrm{~h}$ vs. $12 \mathrm{~h}$ of CCM per day, and was not powered to demon- 
Table 1. Baseline demographics and characteristics. No statistical difference was apparent between the groups.

\begin{tabular}{|c|c|c|c|}
\hline & 5 h group & 12 h group & All \\
\hline $\mathrm{N}$ & 9 & 10 & 19 \\
\hline Age & $62.4 \pm 9.2$ & $63.7 \pm 9.6$ & $63.1 \pm 9.2$ \\
\hline \multicolumn{4}{|l|}{ Gender: } \\
\hline Male & 9 & $9(90 \%)$ & $18(94.7 \%)$ \\
\hline Female & 0 & $1(10 \%)$ & $1(5.3 \%)$ \\
\hline \multicolumn{4}{|l|}{ Cardiomyopathy: } \\
\hline Ischemic & $5(55.6 \%)$ & $3(30 \%)$ & $8(42.1 \%)$ \\
\hline Dilated & $4(44.4 \%)$ & $7(70 \%)$ & $11(57.9 \%)$ \\
\hline \multicolumn{4}{|l|}{ History: } \\
\hline Myocardial infarction & $4(44.4 \%)$ & $3(30 \%)$ & $7(36.8 \%)$ \\
\hline CABG & $2(22.2 \%)$ & $3(30 \%)$ & $5(26.3 \%)$ \\
\hline $\mathrm{PCl}$ & $4(44.4 \%)$ & $5(50 \%)$ & $9(47.4 \%)$ \\
\hline Angina & 0 & 0 & 0 \\
\hline NYHA score & $3.0 \pm 0.0$ & $3.0 \pm 0.0$ & $3.0 \pm 0.0$ \\
\hline MLWHFQ & $52 \pm 20$ & $47 \pm 15$ & $49 \pm 17$ \\
\hline 6MWD & $324 \pm 128(n=9)$ & $333 \pm 107(n=9)$ & $328 \pm 115(n=18)$ \\
\hline Peak $\mathrm{VO}_{2}[\mathrm{~mL} / \mathrm{kg} / \mathrm{min}]$ & $11.8 \pm 2.1$ & $11.8 \pm 2.0$ & $11.8 \pm 2.0$ \\
\hline Ejection fraction & $27 \pm 8$ & $30 \pm 5$ & $29 \pm 6$ \\
\hline LVEDD & $65 \pm 10$ & $59 \pm 11$ & $62 \pm 11$ \\
\hline \multicolumn{4}{|l|}{ Medications: } \\
\hline Beta-blockers & $7(77.8 \%)$ & $7(70 \%)$ & $14(73.7 \%)$ \\
\hline ACE-I & $6(66.7 \%)$ & $6(65 \%)$ & $12(66.1 \%)$ \\
\hline Diuretic & $7(77.8 \%)$ & $8(80 \%)$ & $15(78.9 \%)$ \\
\hline
\end{tabular}

CABG - coronary artery bypass graft; $\mathrm{PCl}$ - percutaneous coronary intervention; NYHA - New York Heart Association classification;

MLWHFQ - Minnesota Living With Heart Failure Questionnaire; 6MWD - 6-min walking distance; peak $\mathrm{VO}_{2}-$ maximal oxygen consumption in cardio-pulmonary stress test; LVEDD — left ventricular end diastolic dimension; ACE-I — angiotensin-converting-enzyme inhibitor

strate statistical significance in safety or efficacy between groups, but to obtain initial assessment of trend, if any.

This was a randomized, double blind, active control study. Descriptive statistics are used to characterize baseline characteristics of the two randomized groups. Differences in changes in efficacy parameters between baseline and 6 months were evaluated based on the two co-primary outcome measures of the mean change in the MLWHFQ score and the mean change in exercise tolerance, as measured by peak $\mathrm{VO}_{2}$ determined during cardiopulmonary exercise stress testing. The results were analyzed by calculating the mean change for each parameter, and the overall level of significance used for this exploratory, feasibility study was 0.05 , calculated by a 2 -sample t-test comparing the 5 vs. 12 CCM hour groups.

Serious adverse events (SAEs) were reported and reviewed for clinical significance.

\section{Results}

Each MLWHFQ, NYHA, peak $\mathrm{VO}_{2}$ and 6MWD co-primary endpoint shows clinical improvement or a trend toward clinical benefit at 24 weeks vs. baseline in patients receiving either $5 \mathrm{~h}$ /day of $\mathrm{CCM}$ or those receiving $12 \mathrm{~h} /$ day stimulation. In the $5 \mathrm{~h}$ /day stimulation group, MLWHFQ showed trend to decrease by $18.5 \pm 26.1$; peak $\mathrm{VO}_{2}$ showed trend to increase by $0.82 \pm 2.81 \mathrm{~mL} / \mathrm{kg} / \mathrm{min}$; and NYHA decreased by $0.88 \pm 0.83(\mathrm{p}=0.02)$. Similarly, in the $12 \mathrm{~h} /$ day stimulation group, MLWHFQ showed trend to decrease by $15.2 \pm 16.9$; peak $\mathrm{VO}_{2}$ showed trend to increase by $2.3 \pm 1.6 \mathrm{~mL} / \mathrm{kg} / \mathrm{min}$; and NYHA decreased by $0.83 \pm 0.75(\mathrm{p}=0.04)$ (Table 2). 6MWD showed similar trend to increase by $32 \mathrm{~m}$ and $29 \mathrm{~m}(\mathrm{p}=\mathrm{NS})$, and there were too little data to determine meaningful changes in EF. There were no significant differences in any of the efficacy measures when comparing the 5 and the 
Table 2. Efficacy data.

\begin{tabular}{|c|c|c|c|c|c|c|}
\hline & & MLWHFQ & $\begin{array}{c}\text { Peak } \mathrm{VO}_{2} \\
{[\mathrm{~mL} \mathrm{O} / \mathrm{kg} / \mathrm{min}]}\end{array}$ & NYHA & $\begin{array}{l}\text { 6MWD } \\
\text { [m] }\end{array}$ & $\begin{array}{l}\text { EF } \\
{[\%]}\end{array}$ \\
\hline \multicolumn{7}{|l|}{5 CCM h/day } \\
\hline \multirow[t]{3}{*}{ Baseline to 24 weeks difference } & Mean & -18.50 & 0.82 & -0.88 & 32.43 & -1.25 \\
\hline & SD & 26.09 & 2.81 & 0.83 & 130.03 & 8.54 \\
\hline & $\mathrm{N}$ & 8 & 5 & 8 & 7 & 4 \\
\hline \multicolumn{7}{|l|}{12 CCM h/day } \\
\hline \multirow[t]{3}{*}{ Baseline to 24 weeks difference } & Mean & -15.17 & 2.30 & -0.83 & 29.60 & 5.75 \\
\hline & SD & 16.94 & 1.61 & 0.75 & 53.49 & 2.99 \\
\hline & $\mathrm{N}$ & 6 & 4 & 6 & 5 & 4 \\
\hline Difference between groups & & 3.33 & 1.48 & 0.04 & -2.83 & 7.00 \\
\hline$P$ & & 0.78 & 0.36 & 0.92 & 0.96 & 0.20 \\
\hline
\end{tabular}

MLWHFQ - Minnesota Living With Heart Failure Questionnaire; peak $\mathrm{VO}_{2}$ - maximal oxygen consumption in cardio-pulmonary stress test; NYHA - New York Heart Association classification; 6MWD - 6-min walking distance; EF — ejection fraction; SD - standard deviation

$12 \mathrm{CCM}$ hours/day groups. Furthermore, in this small cohort, no consistent trends among any efficacy measures were shown to clearly differentiate $5 \mathrm{~h} /$ day or $12 \mathrm{~h} /$ day stimulation groups.

Combining the two study groups revealed an improvement from baseline in two clinical parameters. MLWHFQ improved by $-17.1 \pm 21.9(\mathrm{p}=0.01)$ and NYHA improved by $-0.86 \pm 0.77(\mathrm{p}<0.01)$. Furthermore, trends toward an improvement were observed with peak $\mathrm{VO}_{2}$ (by $1.48 \pm 2.35$ ), $6 \mathrm{MWD}$ (by $31.3 \pm 101.3$ ), and EF (by $2.3 \pm 7.0$ ). The clinical improvement vs. baseline was of similar magnitude to that described previously using $5 \mathrm{~h}$ /day and $7 \mathrm{~h} /$ day of CCM [6-8].

Three subjects were voluntarily withdrawn prior to completing the study for non-medical reasons, and a total of 6 SAEs were reported, none of which was related to either the device or the procedure.

A total of 9 adeverse events were reported in 5 subjects during the study. Of those, 6 were SAEs, reported in 3 subjects, including one death of a subject from pneumonia 125 days post-implant. This death was classified by the investigator as not related to the device or to the procedure. Table 3 summarizes the SAEs. No adverse events were classified by the investigator as related to either the device or the procedure. The reported adverse events are typical of patients with similar degrees of HF. Two patients with SAE were in the $12 \mathrm{~h}$ /day group and 1 patient was in the $5 \mathrm{~h}$ /day group.

There were no device deficiencies reported and blinding by subjects and investigators was maintained throughout the study.
Table 3. Summary of reported serious adverse events.

\begin{tabular}{lcc}
\hline Category of event & $\begin{array}{c}\text { No. of } \\
\text { events }\end{array}$ & $\begin{array}{c}\text { No.of } \\
\text { patients }\end{array}$ \\
\hline Worsening HF & 1 & 1 \\
Progression of CAD & 1 & 1 \\
Arrhythmias (VT, atypical AF) & 2 & 2 \\
$\begin{array}{l}\text { Pneumonia (later leading } \\
\text { to death) }\end{array}$ & 1 & 1 \\
Angina pectoris & 1 & 1 \\
Total & $\mathbf{6}$ & $\mathbf{3}$ \\
\hline
\end{tabular}

HF - heart failure; CAD - coronary artery disease; VT — ventricular tachycardia; AF — atrial fibrillation

\section{Discussion}

The major finding of this study is that both $5 \mathrm{~h} /$ /day and $12 \mathrm{~h}$ /day of CCM are similarly beneficial in terms of quality of life measures and cardiovascular functional improvement at 24 weeks. Furthermore, in this direct comparison between different durations of CCM stimulation, no difference in adverse events was seen. These findings support use of patient-specific programming of duration of CCM stimulation. The experience from past studies and in routine experience (using 3-7 h/day and beyond) combined with the data from this study suggest that any number of CCM h/day in the range of $5 \mathrm{~h}$ to $12 \mathrm{~h}$ may be considered safe and similarly effective, and therefore the physician can apply judgment about tuning the CCM delivery hours per day to each individual patient. 
A very important example of when such tuning of CCM delivery hours per day per patient could be useful is in patients with frequent ectopy. In these cases, the ectopic beats inhibit CCM delivery by the current Optimizer ${ }^{\mathrm{TM}}$ device and may reduce the overall CCM delivery duration. Increasing the average "ON" time per day may compensate for reduced therapy due to beats where CCM was inhibited, and therefore achieves similar overall duration of CCM stimulation as in the absence of ectopy. As experience continues to increase with $\mathrm{CCM}$, it will be of interest to find other subgroups of patients who would benefit from changes in duration of CCM delivery [9].

Despite receiving recommended medical treatment, a significant number of chronic $\mathrm{HF}$ patients remain symptomatic. Nearly $50 \%$ of patients meet the criteria for cardiac resynchronization therapy and of these, as many as $30-50 \%$ are non-responders. Additional treatment modalities known to be clinically effective, such as CCM, may be helpful in these circumstances $[10,11]$.

Within the framework of the small amount of available data, patients appear to experience improvement in their clinical condition, and no note-worthy deterioration in the state of health of subjects was seen in either the $5 \mathrm{~h} /$ day CCM group or the $12 \mathrm{~h} /$ day CCM/group. While no statistical differences were observed between CCM stimulation duration, and together with previously reported studies with CCM therapy, the data suggest that a broad range of daily stimulation durations may be considered safe and effective for CCM therapy.

The safety and efficacy of CCM therapy in HF patients has been shown in a number of studies $[1,5,7,8,12]$. Current evidence indicates that the mechanism of action relates to beneficial modulation of gene expression and protein expression within a few hours of activation [13, 14]. This supports the intermittent application regimen, which also extends battery life. Results of this study showing similar efficacy to prior published work suggest that $5 \mathrm{~h}$ of stimulation per day is sufficient for activating the longer-term genetic and protein remodeling benefit of CCM, and with that to a sustained clinical benefit [15].

\section{Limitations of the study}

This study was not powered to detect a small or modest difference between the $5 \mathrm{~h}$ and $12 \mathrm{~h}$ CCM delivery. However, such a difference is not likely to be present, given the similarity between groups among each of the measured parameters.
In fact, the primary and most secondary measures improved similarly with both durations of stimulation ( $5 \mathrm{~h}$ and $12 \mathrm{~h}$ ) indicating that either duration exceeds the threshold for efficacy. It is not clear whether continued increases in duration of $\mathrm{CCM}$ delivery would be associated with a further improvement in efficacy.

All but one subject enrolled was male and there were no minority subjects. Recruitment of women and minorities is needed to provide a broader diversity-enriched cohort of subjects.

Large absolute differences between $5 \mathrm{~h}$ and $12 \mathrm{~h}$ of stimulation were observed with the assessment of cardiac function (EF). Due to the small sample size and the typical standard deviations in this type of measurement this was not statistically significant. Future studies should examine these endpoints in detail using a larger number of patients.

\section{Conclusions}

In this small cohort, previously reported safety and efficacy of the CCM therapy are equally achieved using duration of CCM delivery of either $5 \mathrm{~h} /$ day or $12 \mathrm{~h} /$ day. These data support the availability of a broader range of CCM delivery durations for optimizing CCM delivery to patients with $\mathrm{HF}$ and reduced $\mathrm{EF}$, expanding opportunities to improve cardiac function and clinical status in patients with moderate to severe HF.

Conflict of interest: This study was sponsored by Impulse Dynamics. Benny Rousso is an employee of Impulse Dynamics. No other author has any conflict of interest.

\section{References}

1. Lyon AR, Samara MA, Feldman DS. Cardiac contractility modulation therapy in advanced systolic heart failure. Nature Rev Cardiol, 2013; 10: 584-598.

2. Borggrefe M, Burkhoff D. Clinical effects of cardiac contractility modulation (CCM) as a treatment for chronic heart failure. Eur J Heart Fail, 2012; 14: 703-712. doi: 10.1093/eurjhf/hfs078.

3. Neelagaru SB, Sanchez JE, Lau SK et al. Nonexcitatory, cardiac contractility modulation electrical impulses: Feasibility study for advanced heart failure in patients with normal QRS duration. Heart Rhythm, 2006; 3: 1140-1147.

4. Stix G, Borggrefe M, Christian W et al. Chronic electrical stimulation during the absolute refractory period of the myocardium improves severe heart failure. Eur Heart J, 2004; 25: 650-655.

5. Kadish A, Nademanee K, Volosin K et al. A randomized controlled trial evaluating the safety and efficacy of cardiac contractility modulation in advanced heart failure. Am Heart J, 2011; 161: 329-337.e1-2.

6. Abraham WT, Nademanee K, Volosin K et al.; FIX-HF-5 Investigators and Coordinators. Subgroup analysis of a randomized 
controlled trial evaluating the safety and efficacy of cardiac contractility modulation in advanced heart failure. J Card Fail, 2011; 17: 710-717.

7. Borggrefe MM, Lawo T, Butter $\mathrm{C}$ et al. Randomized, double blind study of non-excitatory, cardiac contractility modulation electrical impulses for symptomatic heart failure. Eur Heart J, 2008; 29: 1019-1028.

8. Abraham WT, Burkhoff D, Nademanee K et al.; FIX-HF-5 Investigators and Coordinators. A randomized controlled trial to evaluate the safety and efficacy of cardiac contractility modulation in patients with systolic heart failure: Rationale, design, and baseline patient characteristics. Am Heart J, 2008; 156: 641-648.e1.

9. Zhang Q, Chan YS, Liang YJ et al. Comparison of left ventricular reverse remodeling induced by cardiac contractility modulation and cardiac resynchronization therapy in heart failure patients with different QRS durations. Int J Cardiol, 2013; 167: 889-893.

10. McMurray JJ, Adamopoulos S, Anker SD et al. ESC guidelines for the diagnosis and treatment of acute and chronic heart failure 2012: The Task Force for the Diagnosis and Treatment of Acute and Chronic Heart Failure 2012 of the European Society of
Cardiology. Developed in collaboration with the Heart Failure Association (HFA) of the ESC. Eur J Heart Fail, 2012; 14: 803-869.

11. Sandhu R, Bahler RC. Prevalence of QRS prolongation in a community hospital cohort of patients with heart failure and its relation to left ventricular systolic dysfunction. Am J Cardiol, 2004; 93: 244-246.

12. Schau T, Seifert M, Meyhöfer J, Neuss M, Butter C. Long-term outcome of cardiac contractility modulation in patients with severe congestive heart failure. Europace, 2011; 13: 1436-1444.

13. ButterC,RastogiS, MindenHH,MeyhöferJ,BurkhoffD, SabbahHN. Cardiac contractility modulation electrical signals improve myocardial gene expression in patients with heart failure. J Am Coll Cardiol, 2008; 51: 1784-1789.

14. Imai M, Rastogi S, Gupta RC et al. Therapy with cardiac contractility modulation electrical signals improves left ventricular function and remodeling in dogs with chronic heart failure. J Am Coll Cardiol, 2007; 49: 2120-2128.

15. Winter J, Brack KE, Ng GA. Cardiac contractility modulation in the treatment of heart failure: Initial results and unanswered questions. Eur J Heart Fail, 2011; 13: 700-710. 